\title{
Quasi-Monte Carlo methods for derivatives on realised variance of an index under the benchmark approach
}

\author{
J. Baldeaux ${ }^{1} \quad$ L. Chan $^{2} \quad$ E. Platen ${ }^{3}$
}

(Received 30 January 2011; revised 24 August 2011)

\begin{abstract}
We apply quasi-Monte Carlo methods to the pricing of derivatives on realised variance of an index under the benchmark approach. The resulting integration problem is shown to depend on the joint density of the realised variance of the index and the terminal value of the index. Employing a transformation mapping for this joint density to the unit square reduces the difficulty of the resulting integration problem. The quasi-Monte Carlo methods compare favourably to Monte Carlo methods when applied to the given problem.
\end{abstract}

http://anziamj . austms . org . au/ojs/index.php/ANZIAMJ/article/view/3946 gives this article, (C) Austral. Mathematical Soc. 2011. Published August 29, 2011. ISSN 1446-8735. (Print two pages per sheet of paper.) Copies of this article must not be made otherwise available on the internet; instead link directly to this URL for this article. 


\section{Contents}

1 Introduction

C728

2 The stylised minimal market model and options on realised variance

C729

3 Transformation to the unit square

C731

4 Numerical results

C734

5 Conclusion

C738

References

C740

\section{Introduction}

Derivatives on the realised variance of an index, such as the vix, and options on the VIX, as traded on the Chicago Board Options Exchange, have become important risk management tools. The VIX is based on the S\&P 500, a well diversified index, which is interpreted as a proxy for the growth optimal portfolio (GOP). The GOP plays a crucial role in the context of derivative pricing [7]. Here we employ it to price derivatives on realised variance. We find that in order to price derivatives on realised variance, we need the joint distribution of the realised variance of the GOP and its terminal value. We employ a result from Craddock and Lennox [3] to obtain the Laplace transform of the joint distribution. Inverting the Laplace transform, we obtain the joint distribution over $\mathbb{R}^{+} \times \mathbb{R}^{+}$. However, ultimately we wish to employ quasi-Monte Carlo methods to price derivatives on the realised variance of an index. Therefore we need to employ a transformation which maps the joint density to the unit square. In many areas, such as finance and physics, the relevant probability distributions are only known in terms of their Laplace 
transforms. Hence the problem of inverting Laplace transforms and mapping the resulting distributions to the unit square is relevant to many applications and motivates us to study this problem.

The contribution of this article is to explore the relative difficulty of numerical integration problems arising from different transformations. This problem, in the context of quasi-Monte Carlo methods, was also discussed by Kuo et al. [5]. We establish that for both quasi-Monte Carlo and Monte Carlo methods, the difficulty of the integration problem can strongly depend on the transformation, and that quasi-Monte Carlo methods seem to consistently outperform Monte Carlo methods.

Section 2 introduces a model for the GOP, discusses derivative pricing, and considers derivatives on realised variance. Section 3 discusses how to map the joint density to the unit square, and we present results of numerical experiments in Section 4.

\section{The stylised minimal market model and options on realised variance}

We employ the stylised Minimal Market Model (MMM) [7] to model a diversified index, which we interpret as the GOP. We fix a filtered probability space $(\Omega, \mathcal{A}, \underline{\mathcal{A}}, \mathrm{P})$, where $\Omega$ denotes the sample space, $\mathcal{A}$ the sigma-algebra, $\mathrm{P}$ the real-world probability measure, and where the filtration $\underline{\mathcal{A}}=\left(\mathcal{A}_{\mathrm{t}}\right)_{\mathrm{t} \in[0, \infty)}$ is assumed to satisfy the usual conditions [7]. The uncertainty is modeled using a standard Wiener process $\mathrm{W}=\left\{\mathrm{W}_{\mathrm{t}}, \mathrm{t} \in[0, \infty)\right\}$. Furthermore, we introduce a deterministic savings account

$$
\mathrm{dS}_{\mathrm{t}}^{0}=r \mathrm{~S}_{\mathrm{t}}^{0} \mathrm{dt}
$$

for time $t \in[0, \infty)$ with $S_{0}^{0}=1$, where $r$ denotes the constant short rate. We model the index $S_{t}^{\delta_{*}}$ as

$$
S_{t}^{\delta_{*}}=S_{t}^{0} \alpha_{t}^{\delta_{*}} Y_{t}
$$


where $\alpha_{t}^{\delta_{*}}$ is a deterministic function of time, which starts at $\alpha_{0}$ and exhibits exponential growth at the rate $\eta$ :

$$
\alpha_{\mathrm{t}}^{\delta_{*}}=\alpha_{0} \exp \{\eta \mathrm{t}\}
$$

It can be shown that $\alpha_{t}^{\delta_{*}}$ equals the drift of the discounted GOP, and $Y=$ $\left\{Y_{t}, t \in[0, \infty)\right\}$ is a square-root process of dimension four, with stochastic differential equation (SDE)

$$
d Y_{t}=\left(1-\eta Y_{t}\right) d t+\sqrt{Y_{t}} d W_{t} \text { such that } Y_{0}=S_{0}^{\delta_{*}} / \alpha_{0}
$$

The MMM does not admit a risk-neutral martingale measure. However, the GOP is the numéraire when using the real world probability measure $\mathrm{P}$ as pricing measure allowing us to price contingent claims under $P$, using $S^{\delta_{*}}$ as the numéraire. This concept is formally known as real world pricing [7]. Consider a nonnegative contingent claim with payoff $\mathrm{H}$ at time $\mathrm{T}$, assuming that $\mathrm{H}$ is $\mathcal{A}_{\mathrm{T}}$-measurable, and $\mathrm{E}\left(\mathrm{H} / \mathrm{S}_{\mathrm{T}}^{\delta_{*}}\right)<\infty$. Then we define the value at time $\mathrm{t}$ associated with $\mathrm{H}$ by

$$
V_{\mathrm{t}}:=S_{\mathrm{t}}^{\delta_{*}} \mathrm{E}\left(\mathrm{H} / \mathrm{S}_{\mathrm{T}}^{\delta_{*}} \mid \mathcal{A}_{\mathrm{t}}\right)
$$

Since $V_{T}=H, V_{t} / S_{t}^{\delta_{*}}$ forms an $(\mathcal{A}, P)$-martingale. Equation (2) is known as the real world pricing formula and employed to price contingent claims.

We now introduce options on realised variance. Here, the realised variance is defined to be the quadratic variation of the log-index, and we formally compute the quadratic variation of the log-index as

$$
\left[\log \left(S^{\delta_{*}}\right)\right]_{\mathrm{T}}=\int_{0}^{\mathrm{T}} \frac{\mathrm{dt}}{\mathrm{Y}_{\mathrm{t}}} .
$$

In addition, we introduce put options on realised variance. The payoff of such a derivative is

$$
\left(K-\int_{0}^{T} \frac{1}{Y_{t}} d t\right)^{+},
$$


where $\mathrm{K}$ is the strike price. We compute prices using the real world pricing formula, according to which the time $t=0$ price is

$$
S_{0}^{\delta_{*}} E\left[\frac{1}{S_{T}^{\delta_{*}}}\left(K-\int_{0}^{T} \frac{1}{Y_{t}} d t\right)^{+}\right] .
$$

From (1), it suffices to focus on the evaluation of the quantity

$$
E\left[\frac{1}{Y_{T}}\left(K-\int_{0}^{T} \frac{1}{Y_{t}} d t\right)^{+}\right],
$$

which we discuss in the next section. We focus on options on realised variance, as they can be priced explicitly [2], and thus we confirm the accuracy of our results. However, the method discussed in Section 3 is more generally applicable $[2, \S 5]$.

\section{Transformation to the unit square}

This section discusses how to map the joint density of $Y_{T}$ and $\int_{0}^{T} d t / Y_{t}$ to the unit square [5]. We use $f(y, z)$ to denote the joint density of $Y_{T}$ and $\int_{0}^{T} d t / Y_{t}$, where $y$ corresponds to $Y_{\mathrm{T}}$ and $z$ to $\int_{0}^{\mathrm{T}} \mathrm{dt} / \mathrm{Y}_{\mathrm{t}}$. Firstly, we recall Lemma 1 of Craddock and Lennox [3], which gives the Laplace transform of $f(y, z)$. Consequently, we recover the joint density by inverting the Laplace transform. At first sight this seems to amount to inverting a double Laplace transform. However, the technique employed by Craddock and Lennox [3] to compute the double Laplace transform already provides the inversion with respect to one of the parameters.

Lemma 1. The joint Laplace transform of $\mathrm{Y}_{\mathrm{T}}$ and $\int_{0}^{\mathrm{T}} \mathrm{dt} / \mathrm{Y}_{\mathrm{t}}$ is

$$
E\left[\exp \left(-\lambda Y_{T}-\mu \int_{0}^{T} \frac{1}{Y_{t}} d t\right)\right]
$$




$$
\begin{aligned}
= & \frac{\Gamma(3 / 2+v / 2)}{\Gamma(\nu+1)} \beta x^{-1} \exp \left\{\eta\left[T+x-\frac{x}{\tanh (\eta \mathrm{T} / 2)}\right]\right\} \\
& \times \frac{1}{\beta \alpha} \exp \left[\beta^{2} /(2 \alpha)\right] M_{-k, v / 2}\left(\frac{\beta^{2}}{\alpha}\right),
\end{aligned}
$$

where $\alpha=\eta[1+\operatorname{coth}(\eta t / 2)]+\lambda, \beta=(\eta \sqrt{x}) / \sinh (\eta t / 2), v=2 \sqrt{\frac{1}{4}+2 \mu}$, and $\mathrm{M}_{\mathrm{s}, \mathrm{r}}(\mathrm{z})$ denotes the Whittaker function of the first kind. Furthermore, the inverse with respect to $\lambda$ is

$$
\begin{aligned}
p(T, x, y)= & \frac{\eta}{\sinh (\eta T / 2)}\left(\frac{y}{x}\right)^{1 / 2} \\
& \times \exp \left\{\eta\left[T+x-y-\frac{x+y}{\tanh (\eta T / 2)}\right]\right\} I_{v}\left[\frac{2 \eta \sqrt{x y}}{\sinh (\eta T / 2)}\right]
\end{aligned}
$$

where $\mathrm{I}_{v}(\cdot)$ is the modified Bessel function of the first kind.

Consequently, to obtain $f(y, z)$, we only need to invert a one dimensional Laplace transform, which is accomplished via the Euler method described by Abate and Whitt [1]. For the choice of parameters $Y_{0}=1, T=1$ and $\eta=4$, the resulting joint density is shown in Figure 1. Ultimately, we want to employ quasi-Monte Carlo methods to compute (4) and consequently need to map the joint density to the unit square. We follow Kuo et al. [5] in our discussion and recall that $y$ denotes the integration variable corresponding to $Y_{\mathrm{T}}$ and $z$ denotes the integration variable corresponding to $\int_{0}^{\mathrm{T}} \mathrm{dt} / \mathrm{Y}_{\mathrm{t}}$. However, the joint density considered by Kuo et al. [5] was the multivariate normal, which is available explicitly, as opposed to the joint density considered here. We use the substitution

$$
x_{1}=\Psi_{1}(y) \text { and } \quad x_{2}=\Psi_{2}(z),
$$

where $\Psi_{1}$ and $\Psi_{2}$ map the integration variable from $\mathbb{R}^{+}$to $[0,1]$. We consider two choices for $\Psi_{1}$ and $\Psi_{2}$, firstly, the exponential transformation

$$
x_{1}=\Psi_{1}(y)=1-\exp \left\{-\lambda_{1} y\right\} \quad \text { and } \quad x_{2}=\Psi_{2}(z)=1-\exp \left\{-\lambda_{2} z\right\},
$$




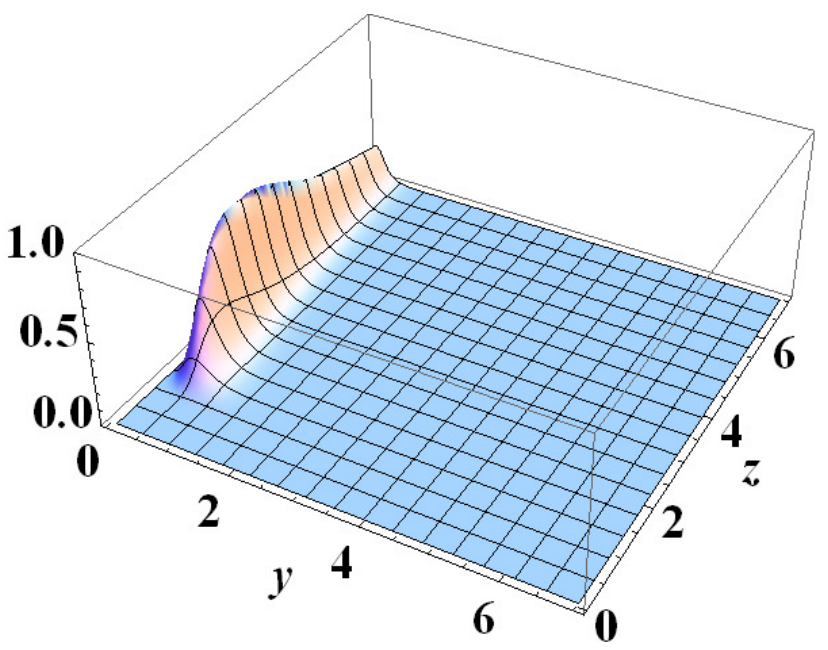

Figure 1: Joint density of $Y_{\mathrm{T}}$ and $\int_{0}^{\mathrm{T}} \mathrm{dt} / \mathrm{Y}_{\mathrm{t}}$ over $\mathbb{R}^{+} \times \mathbb{R}^{+}$.

and secondly the logistic transformation

$$
x_{1}=\Psi_{1}(y)=\frac{\exp \left(y / \lambda_{1}\right)}{1+\exp \left(y / \lambda_{1}\right)} \quad \text { and } \quad x_{2}=\Psi_{2}(z)=\frac{\exp \left(z / \lambda_{2}\right)}{1+\exp \left(z / \lambda_{2}\right)} .
$$

Recall that we use $f(y, z)$ to denote the joint density of $Y_{T}$ and $\int_{0}^{T} d t / Y_{t}$, where $y$ corresponds to $Y_{T}$ and $z$ to $\int_{0}^{T} d t / Y_{t}$, and hence we have the representation

$$
\begin{aligned}
& E\left[\frac{1}{Y_{T}} \mathrm{H}\left(\int_{0}^{T} \frac{d t}{Y_{t}}\right)\right] \\
& =\int_{0}^{\infty} \int_{0}^{\infty} \frac{H(z)}{y} f(y, z) d y d z \\
& =\int_{0}^{1} \int_{0}^{1} \frac{H\left[\Psi_{2}^{-1}\left(x_{2}\right)\right]}{\Psi_{1}^{-1}\left(x_{1}\right)} \mathrm{f}\left[\Psi_{1}^{-1}\left(x_{1}\right), \Psi_{2}^{-1}\left(x_{2}\right)\right] \prod_{j=1}^{2} \frac{1}{\psi_{j}\left[\Psi_{j}^{-1}\left(x_{j}\right)\right]} d x_{j},
\end{aligned}
$$

where $\psi_{1}$ and $\psi_{2}$ denote the densities corresponding to $\Psi_{1}$ and $\Psi_{2}$. We point out two properties of the transformations (7) and (8): firstly, the 
parameters $\lambda_{1}$ and $\lambda_{2}$ are allowed to differ, which turns out to be crucial; and secondly, the transformations are easily understood, which is important, because the joint density is not given explicitly.

Regarding the exponential transformation, we firstly set $\lambda_{1}=1.0$ and $\lambda_{2}=1.0$. From Figure 2(a), it seems advisable to decrease $\lambda_{2}$, which moves the mass of the density towards the centre of the square, see Figure 2(b). Regarding the logistic transformation, we firstly set $\lambda_{1}=1.0$ and $\lambda_{2}=1.0$. From Figure 3(a), it seems advisable to decrease $\lambda_{1}$ and increase $\lambda_{2}$, and we find that the choice $\lambda_{1}=0.25$ and $\lambda_{2}=7.0$ works well, as illustrated in Figure 3(b). Moving the mass of the density towards the center of the square is expected to facilitate the problem. Section 4 confirms that the corresponding standard errors converge at a faster rate. Of course, there exist many ways to make the corresponding numerical integrations more tractable. Once one has found transformations as described above, the difficulties are removed.

\section{Numerical results}

We apply the quasi-Monte Carlo approach to the problem of pricing options on realised variance. A given transformation $\left(\Psi_{1}, \Psi_{2}\right)$ yields

$$
\begin{aligned}
& E\left[\frac{1}{Y_{T}} H\left(\int_{0}^{T} \frac{d t}{Y_{t}}\right)\right] \\
& =\int_{0}^{1} \int_{0}^{1} \frac{H\left[\Psi_{2}^{-1}\left(x_{2}\right)\right]}{\Psi_{1}^{-1}\left(x_{1}\right)} f\left[\Psi_{1}^{-1}\left(x_{1}\right), \Psi_{2}^{-1}\left(x_{2}\right)\right] \prod_{j=1}^{2} \frac{1}{\Psi_{j}\left[\Psi_{j}^{-1}\left(x_{j}\right)\right]} d x_{j} \\
& \approx \frac{1}{N} \sum_{i=1}^{N} \frac{H\left[\Psi_{2}^{-1}\left(x_{i, 2}\right)\right]}{\Psi_{1}^{-1}\left(x_{i, 1}\right)} f\left[\Psi_{1}^{-1}\left(x_{i, 1}\right), \Psi_{2}^{-1}\left(x_{i, 2}\right)\right] \prod_{j=1}^{2} \frac{1}{\psi_{j}\left[\Psi_{j}^{-1}\left(x_{i, j}\right)\right]} \\
& =\frac{1}{N} \sum_{i=1}^{N} g\left(x_{i, 1}, x_{i, 2}\right)
\end{aligned}
$$




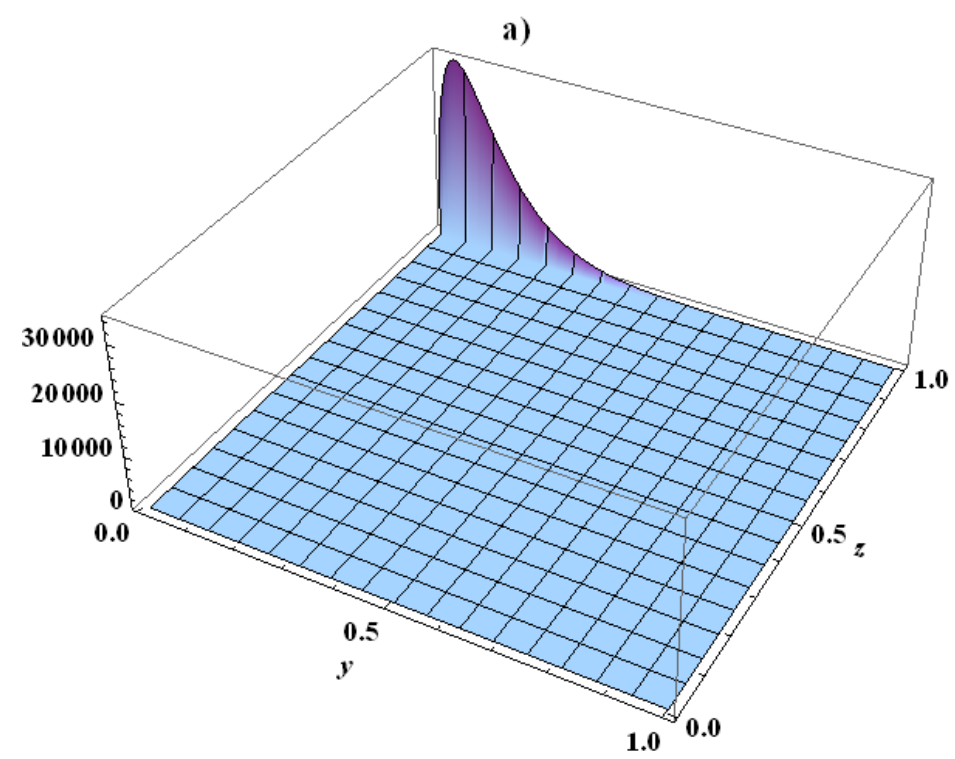

b)

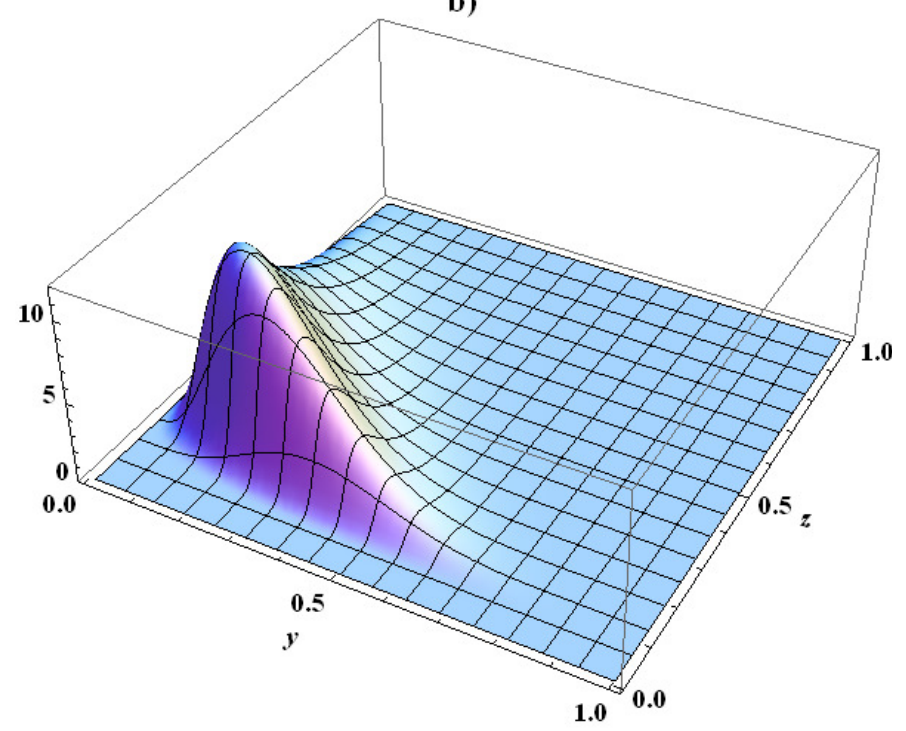

FiguRE 2: Joint density of $Y_{\mathrm{T}}$ and $\int_{0}^{\mathrm{T}} \mathrm{dt} / \mathrm{Y}_{\mathrm{t}}$ : (a) Exponential transformation with $\lambda_{1}=1.0, \lambda_{2}=1.0$; (b) Exponential transformation with $\lambda_{1}=1.0$, $\lambda_{2}=0.1$. 
a)

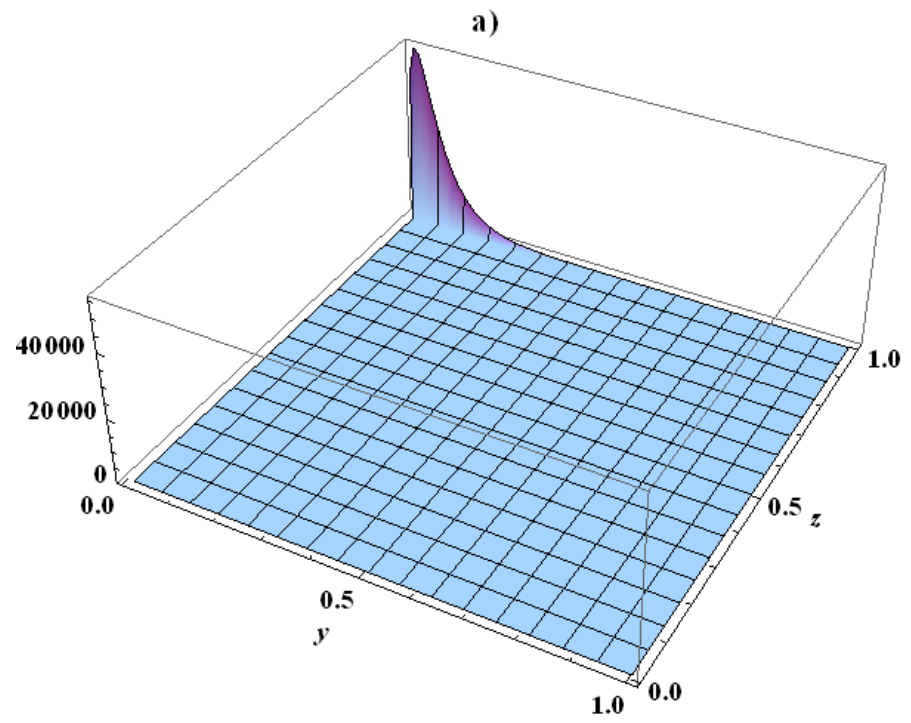

b)

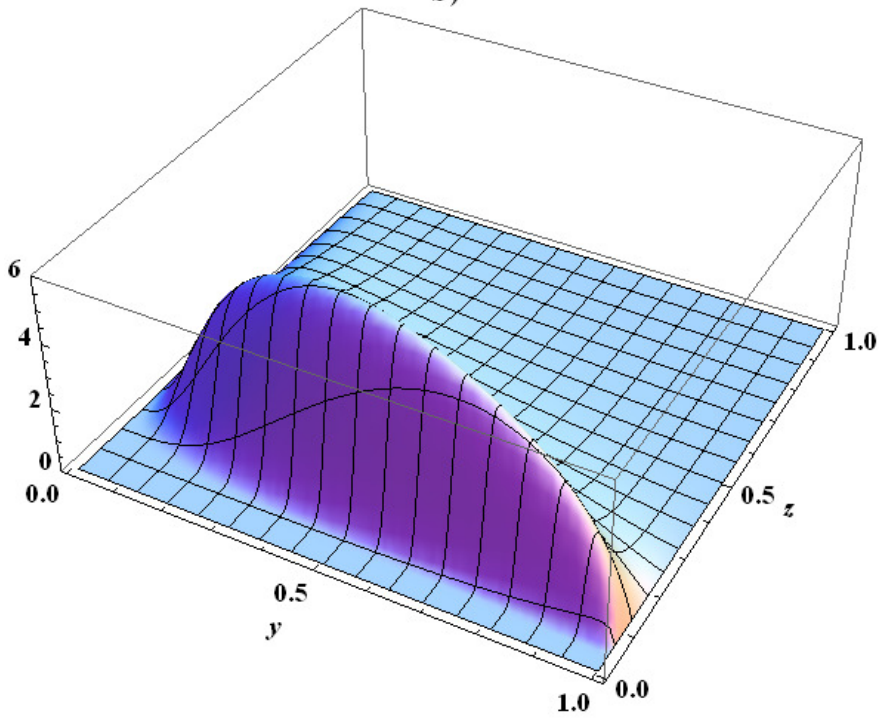

Figure 3: Joint density of $Y_{\mathrm{T}}$ and $\int_{0}^{\mathrm{T}} \mathrm{dt} / \mathrm{Y}_{\mathrm{t}}$ : (a) Logistic transformation with $\lambda_{1}=1.0, \lambda_{2}=1.0$; (b) Logistic transformation with $\lambda_{1}=0.25, \lambda_{2}=7.0$. 
where $\left\{\left(x_{i, 1}, x_{i, 2}\right)\right\}_{i=1}^{N}$ is a two dimensional quasi-Monte Carlo point set and $g\left(x_{i, 1}, x_{i, 2}\right)$ is the obvious function appearing in the above sum. In this article, we base the quasi-Monte Carlo point set on the two dimensional Sobol sequence. Furthermore, we randomize the point set using Owen's scrambling algorithm [6], as implemented by Hong and Hickernell [4]. In this way, we obtain $l$ independent copies of the quasi-Monte Carlo point set, say $\left\{\left(y_{i, 1}^{j}, y_{i, 2}^{j}\right)\right\}_{i=1}^{N}, j=1, \ldots, l$. Consequently, we estimate (4) via

$$
I_{R Q M C}=\frac{1}{l} \sum_{j=1}^{l} I_{j}=\frac{1}{l} \sum_{j=1}^{l} \frac{1}{N} \sum_{i=1}^{N} g\left(y_{i, 1}^{j}, y_{i, 2}^{j}\right),
$$

and estimate standard errors via

$$
\sigma_{\text {RQMC }}=\sqrt{\frac{\sum_{j=1}^{l}\left(I_{j}-I_{R Q M C}\right)^{2}}{l(l-1)}} .
$$

For the purpose of comparison, we also apply the Monte Carlo method; that is, we choose $l N$ independent points $\left\{\left(u_{i, 1}, u_{i, 2}\right)\right\}_{i=1}^{l N}$, uniformly distributed on $[0,1]^{2}$, estimate $(4)$ via

$$
I_{M C}=\frac{1}{l N} \sum_{i=1}^{l N} g\left(u_{i, 1}, u_{i, 2}\right),
$$

and estimate standard errors via

$$
\sigma_{M C}=\sqrt{\frac{\sum_{i=1}^{l N}\left[g\left(u_{i, 1}, u_{i, 2}\right)-I_{M C}\right]^{2}}{l N(l N-1)}} .
$$

Figures 4 and 5 show log-log plots of standard errors against a number of function evaluations. For the quasi-Monte Carlo rules, we use $\mathrm{N}=2^{\mathrm{m}}$ points and compare the results to those achieved with $l \mathrm{~N}=l 2^{\mathrm{m}}$ Monte Carlo points. In all numerical experiments we set $l=30$. These figures show that the transformation does have an impact on the numerical difficulty of the 


\section{standard errors}

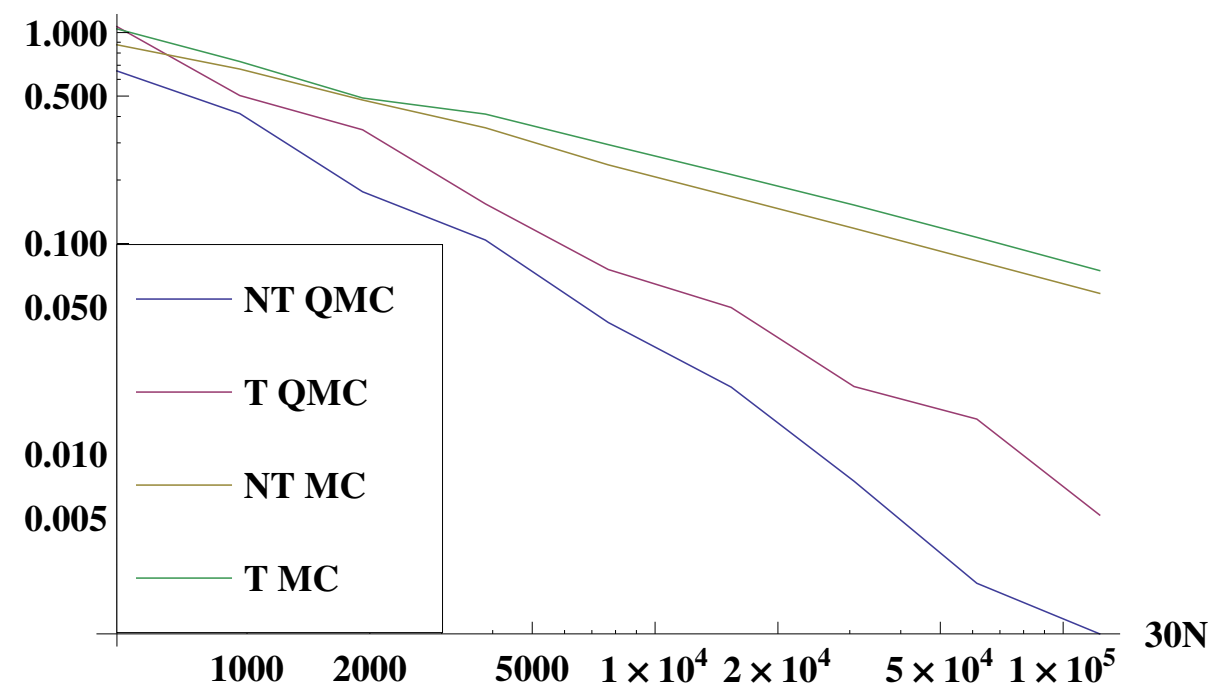

FiguRE 4: Standard errors for the exponential transformation.

integration problem. In the case of the exponential transformation, using the non-trivial (NT) transformation $\lambda_{1}=1.0$ and $\lambda_{2}=0.1$ improves on the trivial $(\mathrm{T})$ choice $\lambda_{1}=1.0$ and $\lambda_{2}=1.0$. For the logistic transformation, using $\lambda_{1}=0.25$ and $\lambda_{2}=7.0(\mathrm{NT})$ improves on $\lambda_{1}=1.0$ and $\lambda_{2}=1.0$ ( $\mathrm{T})$. This observation holds for both quasi-Monte Carlo and Monte Carlo methods, and we also observe that the quasi-Monte Carlo methodology is a significant improvement over Monte Carlo methods. Finally, the standard errors produced by the logistic transform improve on the corresponding standard errors resulting from the exponential transform.

\section{Conclusion}

The problem of mapping joint densities to the unit square arises in important quasi-Monte Carlo applications, such as the pricing of derivatives on the 


\section{standard errors}

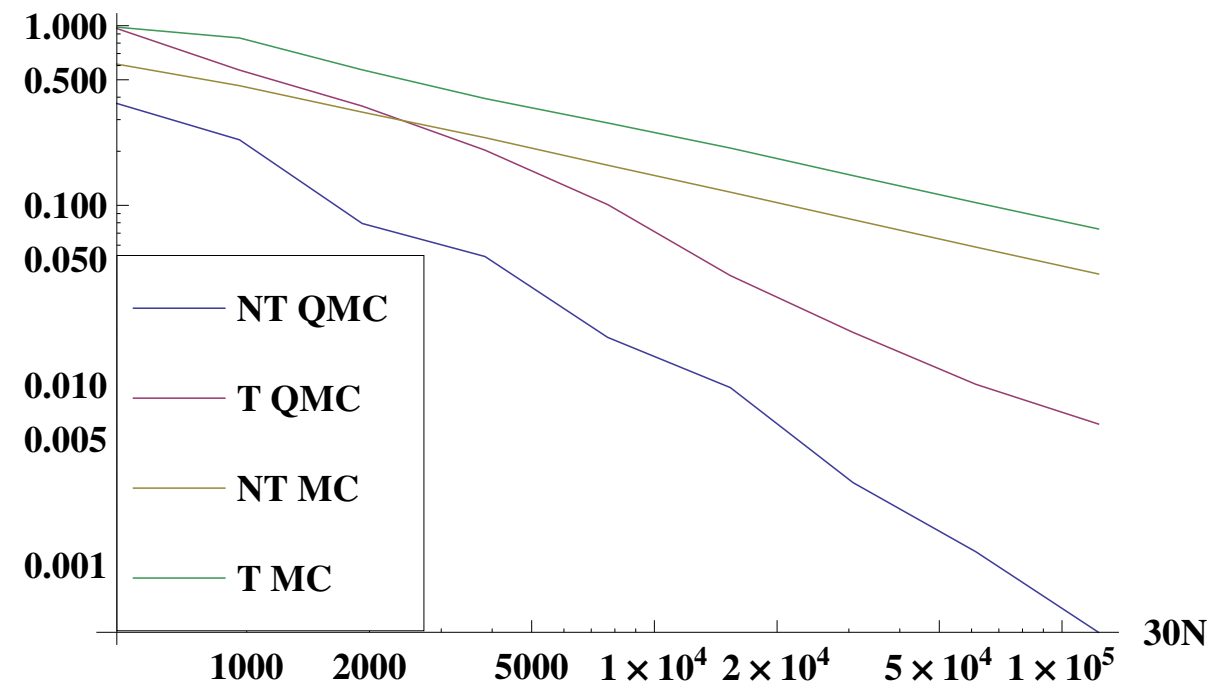

FiguRE 5: Standard errors for the logistic transformation.

realised variance of an index. For the presented two dimensional problem, the resulting joint densities are plotted over the unit square and an appropriate transformation is chosen for the required integration problem.

The challenge is now to generalise the presented methodology to high dimensional problems. The tailoring of the transformations applied in this article may no longer be individually possible. It would be interesting to see how the techniques presented in this article can be applied in a high dimensional context. Quasi-Monte Carlo rules constructed over $\mathbb{R}^{s}$ may be applicable for this type of task. 


\section{References}

[1] J. Abate and W. Whitt, Numerical inversion of Laplace transforms of probability distributions, ORSA J. Comput., 7(1), 36-43, 1995. http: //www. columbia.edu/ ww2040/Fal103/LaplaceInversionJoC95.pdf C732

[2] J. Baldeaux, L. Chan, and E. Platen, Derivatives on realised variance and volatility of an index under the benchmark approach, University of Technology, Sydney, (working paper). C731

[3] M. Craddock and K. Lennox, The calculation of expectations for classes of diffusion processes by Lie symmetry methods, Ann. Appl. Prob., 19(1), 127-157, 2009.

http://arxiv.org/PS_cache/arxiv/pdf/0902/0902.4806v1.pdf C728, C731

[4] H. Hong and F. Hickernell, Algorithm 823: Implementing scrambled digital sequences, ACM Transactions on Mathematical Software, 29(2), 95-109, 2003. C737

[5] F. Y. Kuo, W. T. M. Dunsmuir, I. H. Sloan, M. P. Wand, and R. Womersley, Quasi-Monte Carlo for highly structured generalised response models, Methodology and Comp. Appl. Prob., 10(2), 239-275, 2008. http://www.uow.edu.au/ mwand/publicns/Kuo08.pdf C729, C731, C732

[6] A. B. Owen, Randomly permuted ( $t, m, s)$-nets and ( $t, s)$-sequences, In H. Niederreiter and J. S. Shiue (Eds.), Monte Carlo and quasi-Monte Carlo methods in scientific computing, 299-317, Springer, 1995. C737

[7] E. Platen and D. Heath, A Benchmark Approach to Quantitative Finance, Springer Finance, Springer, 2006. C728, C729, C730 


\section{Author addresses}

1. J. Baldeaux, School of Finance \& Economics, University of Technology Sydney, New South Wales 2007, Australia. mailto: Jan. Baldeaux@uts . edu . au

2. L. Chan, School of Mathematics \& Statistics, University of New South Wales, New South Wales 2052, Australia. mailto: leung. chan@unsw. edu. au

3. E. Platen, School of Finance \& Economics and Department of Mathematical Sciences, University of Technology Sydney, New South Wales 2007, Australia.

mailto:Eckhard.Platen@uts.edu.au 